\title{
RECOGNITION OF N-ACETYL-9-O-ACETYLNEURAMINIC ACID BY BOVINE CORONAVIRUS AND HEMAGGLUTINATING ENCEPHALOMYELITIS VIRUS
}

\author{
Beate Schultze and Georg Herrler \\ Institut für Virologie, Philipps-Universität Marburg \\ Robert-Koch-Str.17, 3550 Marburg, Germany
}

\begin{abstract}
The $S$ protein of hemagglutinating encephalomyelitis virus is shown to be a hemagglutinin requiring $\mathrm{N}$-acetyl-9-O-acetylneuraminic acid as a receptor determinant on the surface of erythrocytes. The ability of bovine coronavirus to recognize 9-O-acetylated sialic acid was used to establish a binding assay for the detection of glycoproteins containing this type of sugar. The assay is very fast, because it uses the acetylesterase of the viral HE protein to localize bound virus.
\end{abstract}

\section{INTRODUCTION}

Bovine coronavirus (BCV) has been known for some years to require 9-O-acetylated sialic acid on the cell surface for agglutination of erythrocytes $(1,2)$. Recently, it has been shown that BCV uses $\mathrm{N}$-acetyl-9-O-acetylneuraminic acid as a receptor determinant also on cultured cells to initiate infection (3). The binding of BCV to receptors containing 9$\mathrm{O}$-acetylated sialic acid can be mediated by two viral surface proteins. The HE protein, which also has acetylesterase activity, is able to agglutinate mouse or rat erythrocytes $(4,5)$; these cells contain a high proportion of $\mathrm{N}$-acetyl-9-O-acetylneuraminic acid $(50 \%$ 
and more) among their surface-bound sialic acids. The $S$ protein has recently been shown to recognize 9-0-acetylated sialic acid, too (5). In fact, it requires fewer receptors on the surface of erythrocytes for agglutination than does the HE protein. Therefore, the $S$ protein is the major hemagglutinin of $\mathrm{BCV}$.

Here we report that hemagglutinating activity can be assigned not only to the $S$ protein of $\mathrm{BCV}$, but also to the $S$ protein of hemagglutinating encephalomyelitis virus (HEV). In addition we show that the activities of the surface proteins $\mathrm{S}$ (binding to $\mathrm{N}$ acetyl-9-O-acetylneuraminic acid) and HE (acetylesterase) can be used in a sensitive assay for the detection of glycoproteins containing 9-O-acetylated sialic acid.

\section{MATERIALS AND METHODS}

Viruses and Cells. Strain L-9 of BCV and strain NT-9 of HEV were grown in MDCK I cells as described previously (2).

Isolation of Viral Glycoproteins. The $S$ and HE protein of HEV were isolated as reported recently (6).

Hemagglutination Assay. The hemagglutinating activity of virus and isolated glycoproteins was determined according to published procedures $(2,5,6)$.

Binding Assay. The immobilization of glycoproteins on nitrocellulose and the binding of $\mathrm{BCV}$ have been described recently (5). Bound virus was detected according to the method described for influenza $\mathrm{C}$ virus by Zimmer et al. (7).

\section{RESULTS AND DISCUSSION}

\section{The S Protein of BCV and HEV has hemagglutinating Activity}

The isolation of the viral surface proteins $S$ and $\mathrm{HE}$ has been described for both BCV and HEV (6). Following removal of the detergent octylglucoside by dialysis, the $S$ protein was analyzed for hemagglutinating acitivity. As shown in Table 1, the same result was obtained with HEV as has been reported previously for BCV. Erythrocytes from adult chickens were agglutinated by virus and by isolated S, but not by HE Protein. No hemagglutination was observed with erythrocytes from one-day old chicken. The latter cells lack 9-O-acetylated sialic acid - in contrast to erythrocytes from adult chicken (8). The 
Table 1. Hemagglutinating activity of $\mathrm{BCV}, \mathrm{HEV}$, and the isolated proteins $\mathrm{S}$ and $\mathrm{HE}$.

$\begin{aligned} & \text { hemagglutinating activity }(\mathrm{HA}-\mathrm{units} / \mathrm{ml}) \\ & \text { adult chicken }\end{aligned}$
$\begin{gathered}\text { with erythrocytes from } \\ \text { one-day old chicken }\end{gathered}$
BCV

\begin{tabular}{lcccc} 
virus & 256 & 128 & $<2$ & $<2$ \\
S protein & 1024 & 256 & $<2$ & $<2$ \\
& $<2$ & $<2$ & $<2$ & $<2$ \\
\hline
\end{tabular}

result from Table 1 indicates that isolated $S$ protein of $\mathrm{HEV}$ uses the same type of receptors as does the $\mathrm{S}$ protein of $\mathrm{BCV}$, i.e. it is dependent on the presence of $\mathrm{N}$-acetyl-9-Oacetylneuraminic acid.

\section{Use of BCV for the Detection of Glycoproteins containing N-acetyl-9-O-acetylneuraminic acid}

We have previously reported that BCV can be used as a lectin to identify glycoproteins which contain N-acetyl-9-O-acetylneuraminic acid (5). For this purpose, glycoproteins were immobilized by transfer from a SDS-polyacrylamide gel to nitrocellulose. After incubation with $\mathrm{BCV}$, virus which had bound to individual proteins, was identified by a enzyme-linked immunoreaction. This detection system is rather timeconsuming, because it requires several consecutive incubations (first antibody, second antibody, enzyme, substrate) with intermittent washings. This assay was modified such that the acetylesterase of BCV was used to detect bound virus as has been described recently for influenza $\mathrm{C}$ virus (7). The principle is shown in Fig.1. After BCV has bound to the immobilized glycoproteins, the substrate $\alpha$-naphthylacetate is added. The acetylesterase activity of $\mathrm{HE}$ cleaves the substrate releasing $\alpha$-naphthol. The latter compound reacts with Fast Red, which is included in the substrate solution, resulting in a colored insoluble complex. The colored precipitate indicates the presence of bound virus 
$\stackrel{9}{9}$

$\downarrow+$ virus

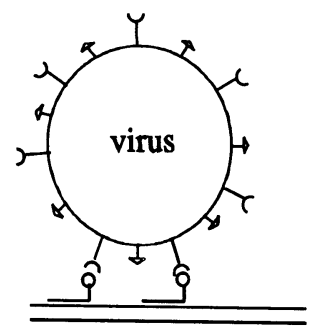

$\perp+\alpha$-naphthyl acetate

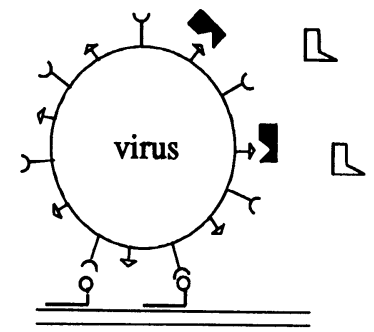

1

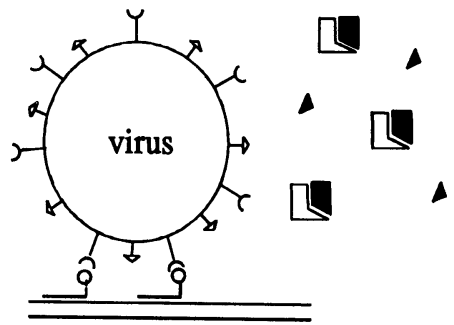

9 sialic acid-containing glycoconjugate

个 HE protein

Y S protein

$\alpha$-naphthyl acetate

$\alpha$-naphthol

acetate

Fast Red

coloured complex (insoluble)

Figure 1. Schematic illustration of the binding assay with BCV for the detection of glycoproteins containing $\mathrm{N}$-acetyl-9-O-acetylneuraminic acid. 
and therefore, the presence of glycoproteins containing 9-0-acetylated sialic acid.

This assay was applied to the glycoproteins of rat serum which are known to contain $\mathrm{N}$-acetyl-9-O-acetylneuraminic acid. As shown in Fig. 2, the staining pattern indicates that $\mathrm{BCV}$ bound to many bands. In order to show that the binding of BCV to these proteins is due to the presence of N-acetyl-9-O-acetylneuraminic acid, a control sample was pretreated with sodium hydroxide. Under these alkaline conditions $\mathrm{O}$-acetyl groups

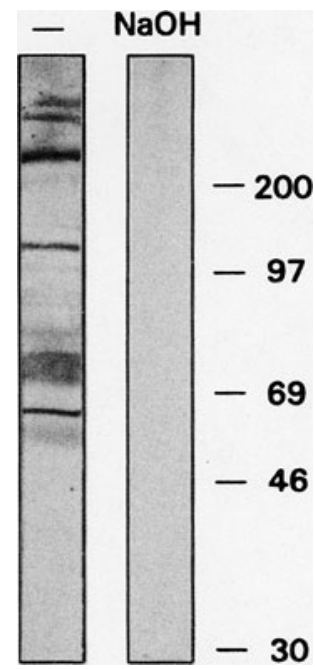

Figure 2. Detection of glycoproteins containing $\mathrm{N}$-acetyl-9-0-acetylneuraminic acid in rat serum after immobilization of the proteins on nitrocellulose.

of sialic acid are released while the remainder of the oligosacharides is unaffected. The lack of staining of rat serum proteins after incubation with sodium hydroxide indicates that bands revealed by the binding assay with BCV are actually due to the presence of $\mathrm{N}$ acetyl-9-O-acetylneuraminic acid.

By applying this assay to cell surface proteins it should be possible in the future to identify potential receptors for $\mathrm{BCV}$ and related coronaviruses among cell surface proteins.

\section{ACKNOWLEDGMENTS}

We thank Gert Zimmer for help with the binding assay. This work was supported by Deutsche Forschungsgemeinschaft (He 1168/2-2). 


\section{REFERENCES}

1. R. Vlasak, W. Luytjes, W. Spaan, and P. Palese, P., Proc.Natl.Acad.Sci.USA 85:4526 (1988).

2. B. Schultze, H.-J. Gross, R. Brossmer, H.-D. Klenk, and G. Herrler, Virus Res. 16:185 (1990).

3. B. Schultze, and G. Herrler, 1992, J.Gen.Virol. 73:901 (1992).

4. B. King, B.J. Potts, and D.A. Brian, Virus Res. 2:53 (1985).

5. B. Schultze, H.-J. Gross, R. Brossmer, and G. Herrler, J.Virol. 65:6232 (1991).

6. B. Schultze, K. Wahn, H.-D. Klenk, and G. Herrler, 1991a, Virology 180:221 (1991).

7. G. Zimmer, G. Reuter, and R. Schauer, Eur.J.Biochem. 204:209 (1992).

8. G. Herrler, G. Reuter, R. Rott, H.-D. Klenk, and R. Schauer, Biol.Chem.Hoppe-Seyler 368:451 (1987). 